Original Research Paper

\title{
Evaluation of the Tunnel Face Stability through a Ground Stress Analysis with a Hemispherical Geometry Approximation
}

\author{
Pierpaolo Oreste \\ Department of Environmental, Land and Infrastructural Engineering, Politecnico di Torino (Italy) Corso Duca degli Abruzzi \\ 24, I-10129 Torino, Italy
}

Article history

Received: 2014-11-12

Revised: 2014-11-16

Accepted: 2014-12-10

E-mail: pierpaolo.oreste@polito.it

\begin{abstract}
The evaluation of the stability of the excavation face is an important aspect in the design of a tunnel. When it is not possible to ensure excavation face stability in natural conditions, it is in fact necessary to intervene with remarkably costly reinforcement operations. The analysis of the stability conditions of an excavation face can be conducted, with a certain degree of detail, through numerical modelling. Simplified analytical models exist for shallow tunnels, but fewer are available for deep tunnels. One of the analytical methods most commonly used in the study of the stress conditions at the excavation face in deep tunnels is described in this study: The convergence-confinement method adapted to a spherical geometry. This method has here been extended to rock masses, which present a more complex rupture criterion (curved and not simply linear) than that of soils. The presented solution is of a finite difference numerical type. An extensive parametric analysis conducted on soils and rock masses has led to the estimation of the maximum lithostatic stress that still foresees the absence of a plastic zone around the hemisphere, which has been used to represent the excavation face. Therefore, this study makes it possible to obtain a preliminary estimation of the maximum depth of a tunnel in a certain type of soil or in a rock mass in which it is still possible to advance without the necessity of excavation face reinforcement operations. However, a more detailed and reliable analysis still requires more sophisticated instruments, such as numerical modelling.
\end{abstract}

Keywords: Tunnel Facestability, Convergence-Confinement Method, Elasto-Plastic Behaviour, Deep Circulartunnel, Stress Strain Evaluation, Plastic Zones, Tunnel Collapse, Hemispherical Approximation

\section{Introduction}

The stability of an excavation face is one of the most important static problems in the tunneling field (Anagnostou and Kovari, 1996; Lunardi and Bindi, 2004; Lunardi, 2008; Oreste, 2009; Oreste and Dias, 2012; Oreste, 2013). A tunnel usually collapses nowadays because of the collapse of the excavation face. This phenomenon can involve both tunnels excavated in soil and those excavated in rock masses. Moreover, tunnel collapses have been recorded in both shallow and deep tunnels.

It is therefore necessary to be able to know the excavation face stability conditions during the design phase and in particular to know whether it will be necessary to carry out reinforcement of the excavation face or not. An excavation face is reinforced through the introduction of cemented fiberglass dowels into boreholes positioned in a direction parallel to the tunnel axis. This kind of intervention, which may require an elevated number of dowels (up to 1 for each square meter of excavation face), could be very costly and should therefore only be used when instability of the excavation face occurs in natural conditions, that is, without reinforcement interventions.

The stability of an excavation face can be evaluated through numerical calculation methods (with axialsymmetric two-dimensional or three-dimensional geometries) (Dias et al., 1997; Dias and Kastner, 2005; $\mathrm{Ng}$ and Lee, 2002; Wong et al., 2004; Yoo, 2002; Yoo and Shin, 2003). Numerical methods allow to obtain a very detailed evaluation of the stress and strain conditions in the soil or in the rock mass and in the foreseen support structures and reinforcement elements (Do et al., 2013; 
2014a; 2014b). Simplified analytical methods are widely used in tunneling because they are quicker for the calculation and interpretation of the results (Oreste, 2007; Do et al., 2014c) and; moreover, they are particularly suitable to develop probabilistic calculations (Oreste, 2005a) and back-analysis studies (Oreste, 2005b) due to their calculation speed. Some examples have been developed for the analysis of static conditions in shallow tunnels (Oreste, 2009; Oreste and Dias, 2012). However, specific calculation methods for the evaluation of face stability are not so common for deep tunnels (greater tunnel axis depths than 10-12 times the radius) (Leca and Panet, 1988; Leca and Dormieux, 1990; Kirsch, 2010; Kamata and Mashimo, 2003; Wong et al., 2000; Boldini et al., 2004).

In the past, some authors adopted the convergenceconfinement method, adapted to a spherical geometry (Lembo-Fazio and Ribacchi, 1986; Yu, 2000), in order to be able to study the stability conditions of an excavation face, using the Mohr-Coulomb linear rupture criterion.

A detailed analysis of the stress state ahead of the excavation face is given in this study for a deep tunnel, utilizing the convergence-confinement method adapted to a spherical geometry, but also extending it to rock masses, for which a non-linear rupture criterion is valid. Extension of the method to rock masses has required the adoption of a simple solution of a finite difference numerical type.

The results of an extensive parametric analysis are then presented in order to be able to evaluate the static conditions of the excavation face under the different geometric and geotechnical conditions that can be encountered during the excavation of a tunnel.

\section{Materials and Methods}

The stability of the excavation face in a deep tunnel can be assessed, with a certain degree of approximation, considering a hemisphere with a radius equal to the radius of the tunnel, positioned in the same way as shown in Fig. 1. Using this simplified hypothesis, it is possible to easily proceed with the evaluation of the stresses that develop in the ground ahead of the excavation face. Then, on the basis of the stress analysis, it is useful to verify whether the ground shows complete elastic behavior or whether there is a plastic portion (plastic cortex) in contact with the hemispheric surface that represents the excavation face.

The presence of a plastic zone ahead of the excavation face generally indicates instability of various degrees (which depends on the thickness of the plastic zone), with the consequent possibility of the excavation face collapsing or at least showing the breakage of portions of material of various sizes.

An approximation of the excavation face with a hemispheric geometry makes it possible to proceed with the evaluation of the radial and circumferential stresses as it is possible to take advantage of the spherical symmetry (Fig. 2).

Among the various hypotheses of the convergenceconfinement method utilized for the analysis of the stresses in the ground (Rechsteiner and Lombardi, 1974; Ribacchi and Riccioni, 1977; Panet and Guenot, 1982; AFTES, 1993; Panet, 1995; AFTES, 2001; Oreste, 2003a; 2003b; Osgoui and Oreste, 2010; Oreste, 2008; Wong et al., 2006), there is that of homogeneous and isotropic lithostatic stresses: The stresses existing in the medium before the creation of the hemispheric void are equal to $\mathrm{p}_{0}$ in all directions. On the basis of this hypothesis, it is possible to describe the radial stresses $\sigma_{\mathrm{r}}$ and the circumferential stresses $\sigma_{\vartheta}$, under elastic behavior conditions of the medium around a sphere, using the following simple equations (Lembo-Fazio and Ribacchi, 1986), when a nil radial pressure is applied to the hemispherical surface:

$$
\begin{aligned}
& \sigma_{\theta}=p_{0} \cdot\left(1+\frac{1}{2} \cdot \frac{R^{3}}{r^{3}}\right) \\
& \sigma_{r}=p_{0} \cdot\left(1-\frac{R^{3}}{r^{3}}\right)
\end{aligned}
$$

Where:

$\mathrm{p}_{0}=$ The lithostatic stress that exists at a tunnel depth;

$\mathrm{R}=$ The radius of the sphere;

$r=$ The distance from the center of the sphere.

Legend: (R): Radius of the hemisphere and of the tunnel; $\alpha$ and $\delta$ : Polar coordinate angles. The axis of the tunnel coincides with the $\mathrm{x}$ axis of the Cartesian system. The radial stresses $\sigma_{\mathrm{r}}$ are those that act in the direction of the connection to the center of the sphere, while the circumferential stresses $\sigma_{\vartheta}$ are directed perpendicularly to the direction of the connection to the center of the sphere.

In the presence of a plastic zone around the hemisphere, whose extension (if it exists) is from $\mathrm{R}$ to the plastic radius $R_{\mathrm{pl}}$, the medium beyond the plastic zone $\left(r>R_{p l}\right)$ has elastic behavior and the radial and circumferential stresses are expressed by the following equations (Lembo-Fazio and Ribacchi, 1986):

$$
\begin{aligned}
& \sigma_{\theta}=p_{0}+\frac{1}{2} \cdot\left(p_{0}-\sigma_{R p l}\right) \cdot \frac{R_{p l}^{3}}{r^{3}} \\
& \sigma_{r}=p_{0}-\left(p_{0}-\sigma_{R p l}\right) \cdot \frac{R_{p l}^{3}}{r^{3}}
\end{aligned}
$$

Where:

$\sigma_{\mathrm{Rpl}}=$ The radial stress at the plastic radius $\mathrm{R}_{\mathrm{pl}}$. 


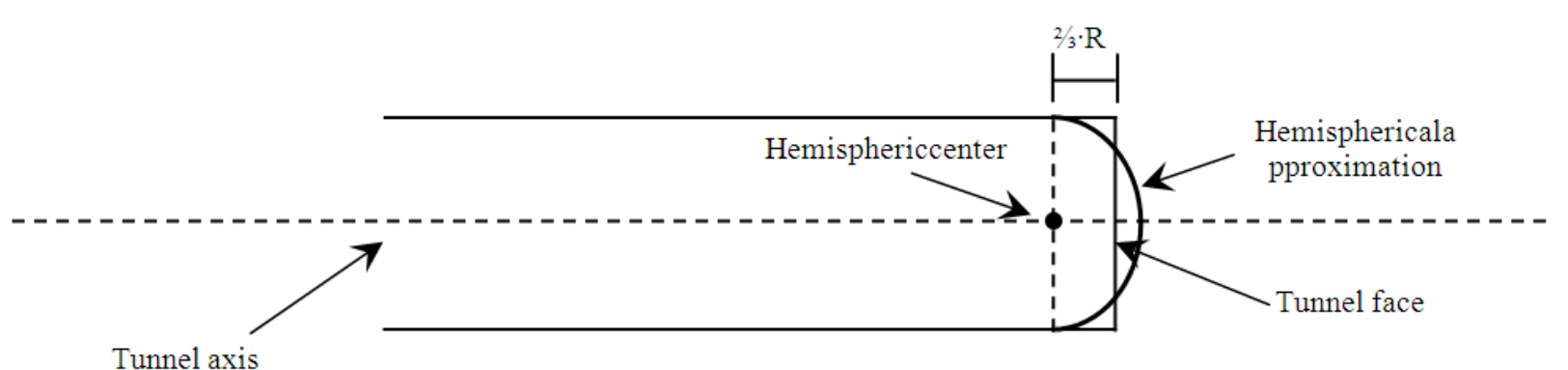

Fig. 1. Approximation of the excavation face with a hemispherical geometry with a radius equal to the tunnel radius. The position of the center of the hemisphere is moved back by $2 / 3 \cdot \mathrm{R}$ with respect to the excavation face, where $\mathrm{R}$ stands for the radius of the tunnel

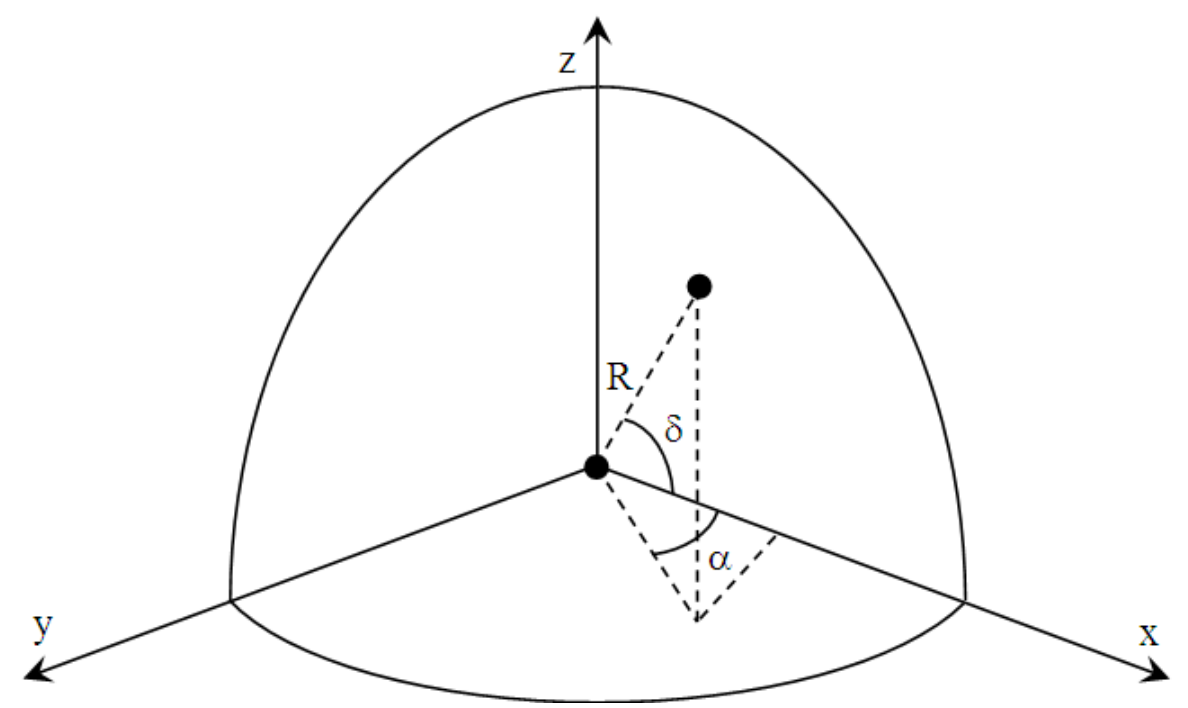

Fig. 2. Spherical symmetry used in the study to approximate the stress conditions ahead of the excavation face

The Mohr-Coulomb linear strength criterion, whose expression, in terms of principle stresses, has the following form, is generally used for soil:

$\sigma_{1, \lim }=\sigma_{3} \cdot \frac{1+\operatorname{sen} \varphi}{1-\operatorname{sen} \varphi}+\frac{2 \cdot c \cdot \cos \varphi}{1-\operatorname{sen} \varphi}$

Where:

$\sigma_{1, \lim }=$ The maximum principle stress upon breaking of the rock mass;

$\sigma_{3}=$ The minimum principle stress (confinement);

$\mathrm{c} \quad=$ The soil cohesion;

$\varphi \quad=$ The soil friction angle.

The by now universally used strength criterion for rock masses is that of Hoek and Brown, in its updated version (Hoek and Brown, 1980; Carranza-Torres and Fairhurst, 2000; Hoek et al., 2002):

$\sigma_{1, \lim }=\sigma_{3}+\sigma_{c i} \cdot\left(m_{b} \cdot \frac{\sigma_{3}}{\sigma_{c i}}+s\right)^{a}$
Where:

$\sigma_{\mathrm{ci}}=$ The uniaxial compression strength of the intact rock;

$\mathrm{m}_{\mathrm{b}}$ and $\mathrm{s}=$ The strength parameters, which depend on the Geological Strength Index (GSI) (Marinos et al., 2005; Hoek et al., 2013; Marinos and Hoek, 2000) and on the D parameter (Ribacchi and Riccioni, 1977): $m_{b}=m_{i} \cdot e^{\left(\frac{G S I-100}{28-14 \cdot D}\right)} ; s=e^{\left(\frac{G S I-100}{9-3 \cdot D}\right)}$

$\mathrm{D}=\mathrm{A}$ parameter that varies between 0 and 1 , which considers the disturbance of the rock mass due to the excavation operations ( $\mathrm{D}=0$ for non-disturbed mass; $\mathrm{D}=1$ for intensely disturbed mass);

$\mathrm{m}_{\mathrm{i}} \quad=$ A strength parameter that refers to intact rock and which depends on the typology of the rock (Hoek et al., 2002);

a $\quad=$ The exponent that is present in Equation 6: $a=0.5+\frac{1}{6} \cdot\left(e^{-\frac{G S I}{15}}-e^{-\frac{20}{3}}\right)$. 
The above mentioned strength criteria can be applied to both the peak conditions (elastic behavior limit conditions) and to the residual conditions (conditions in the plastic field) (the subscript $\mathrm{p}$ refers to the peak conditions, while the subscript res refers to the residual conditions):

$\sigma_{1, \lim }=\sigma_{3} \cdot \frac{1+\operatorname{sen} \varphi_{p}}{1-\operatorname{sen} \varphi_{p}}+\frac{2 \cdot c_{p} \cdot \cos \varphi_{p}}{1-\operatorname{sen} \varphi_{p}}$

Mohr-Coulomb strength criterion (peak condition)

$\sigma_{1, \lim }=\sigma_{3} \cdot \frac{1+\operatorname{sen} \varphi_{r e s}}{1-\operatorname{sen} \varphi_{r e s}}+\frac{2 \cdot c_{r e s} \cdot \cos \varphi_{r e s}}{1-\operatorname{sen} \varphi_{r e s}}$

Mohr-Coulomb strength criterion (residual condition)

$\sigma_{1, \lim }=\sigma_{3}+\sigma_{c i} \cdot\left(m_{b p} \cdot \frac{\sigma_{3}}{\sigma_{c i}}+s_{p}\right)^{a_{p}}$

Hoek-Brown strength criterion (peak condition)

$\sigma_{1, \mathrm{lim}}=\sigma_{3}+\sigma_{c i} \cdot\left(m_{b r e s} \cdot \frac{\sigma_{3}}{\sigma_{c i}}+s_{r e s}\right)^{a_{r e s}}$

Hoek-Brown strength criterion (residual condition)

The radial stress $\sigma_{R p l}$ at the plastic radius $\left(r=R_{p l}\right)$ is obtained by introducing the equivalences of Equation 4 (valid for the elastic behavior zone) with the peak strength criterion (Equations 7a and 8a):

$\frac{3}{2} \cdot\left(p_{0}-\sigma_{R p l}\right)=\sigma_{R p l} \cdot \frac{2 \cdot \operatorname{sen} \varphi_{p}}{1-\operatorname{sen} \varphi_{p}}+\frac{2 \cdot c_{p} \cdot \cos \varphi_{p}}{1-\operatorname{sen} \varphi_{p}}$

For Mohr-Coulomb criterion

$\frac{3}{2} \cdot\left(p_{0}-\sigma_{R p l}\right)=\sigma_{c i} \cdot\left(m_{b p} \cdot \frac{\sigma_{R p l}}{\sigma_{c i}}+s_{p}\right)^{a_{p}}$

For Hoek-Brown criterion

From which:

$\sigma_{R p l}=\frac{\frac{3}{2} \cdot p_{0}-\frac{2 \cdot c_{p} \cdot \cos \varphi_{p}}{1-\operatorname{sen} \varphi_{p}}}{\left(\frac{2 \cdot \operatorname{sen} \varphi_{p}}{1-\operatorname{sen} \varphi_{p}}+\frac{3}{2}\right)}$

For Mohr-Coulomb criterion

$p_{0}-\sigma_{R p l}=\frac{2}{3} \cdot \sigma_{c i} \cdot\left(m_{b p} \cdot \frac{\sigma_{R p l}}{\sigma_{c i}}+s_{p}\right)^{a_{p}}$

For Hoek-Brown criterion

Equation 10 can be resolved directly (if the cohesion and friction angles in peak conditions are known as well as the lithostatic stress $\mathrm{p}_{0}$ ), Equation $10 \mathrm{~b}$ is resolved numerically.
If $\sigma_{\mathrm{Rpl}}$ is below zero, no plastic zone will form around the sphere and the entire medium will have elastic behavior. If, instead, $\sigma_{\mathrm{Rpl}}$ is above zero, a plastic zone will form (between $r=R$ and $r=R_{p l}$ ), inside of which the radial stresses will reduce from $\sigma_{\mathrm{r}}=\sigma_{\mathrm{Rpl}}$ for $\mathrm{r}$ $=\mathrm{R}_{\mathrm{pl}}$ to $\sigma_{\mathrm{r}}=0$ for $\mathrm{r}=\mathrm{R}$.

The trend of the radial stresses in the plastic zone is dictated by the following differential equation (LemboFazio and Ribacchi, 1986):

$\frac{d \sigma_{r}}{d r}=2 \cdot \frac{\sigma_{\theta}-\sigma_{r}}{r}$

By substituting Equations $7 \mathrm{~b}$ or $8 \mathrm{~b}$ (strength criterion of the medium in residual conditions, which is valid inside the plastic zone) in Equation 11 and knowing that the radial stresses are the minimum principle stresses and the circumferential ones are the maximum principle stresses, we obtain:

For Mohr-Coulomb strength criterion (Equations 12-15):

$\frac{d \sigma_{r}}{d r}=2 \cdot \frac{\sigma_{r} \cdot \frac{2 \cdot \operatorname{sen} \varphi_{r e s}}{1-\operatorname{sen} \varphi_{\text {res }}}+\frac{2 \cdot c_{r e s} \cdot \cos \varphi_{r e s}}{1-\operatorname{sen} \varphi_{\text {res }}}}{r}$

From which, by proceeding with the integration between the radius of the sphere and the plastic radius, we obtain:

$\int_{0}^{\sigma_{R p l}} \frac{d \sigma_{r}}{A \cdot \sigma_{r}+B}=\int_{R}^{R_{p l}} \frac{d r}{r}$

Where:

$$
A=\frac{4 \cdot \operatorname{sen} \varphi_{\text {res }}}{1-\operatorname{sen} \varphi_{\text {res }}}
$$

And:

$$
B=\frac{4 \cdot c_{r e s} \cdot \cos \varphi_{r e s}}{1-\operatorname{sen} \varphi_{r e s}}
$$

Finally, by resolving the integral of Equation 13, the searched for plastic radius is obtained:

$$
R_{p l}=R \cdot\left(\frac{A \cdot \sigma_{R p l}+B}{B}\right)^{\frac{1}{A}}
$$

Which, re-written in extended form, becomes:

$$
\begin{aligned}
& R_{p l}= \\
& R \cdot\left\{1+\frac{\operatorname{sen} \varphi_{r e s} \cdot\left[\frac{3}{2} \cdot p_{0} \cdot\left(1-\operatorname{sen} \varphi_{p}\right)-2 \cdot c_{p} \cdot \cos \varphi_{p}\right]}{c_{r e s} \cdot \cos \varphi_{r e s} \cdot\left[2 \cdot \operatorname{sen} \varphi_{p}+\frac{3}{2} \cdot\left(1-\operatorname{sen} \varphi_{p}\right)\right]}\right\}
\end{aligned}
$$


For Hoek-Brown strength criterion (Equations 16-17):

$$
\frac{d \sigma_{r}}{d r}=2 \cdot \frac{\sigma_{c i} \cdot\left(m_{b r e s} \cdot \frac{\sigma_{r}}{\sigma_{c i}}+s_{\text {res }}\right)^{a_{r e s}}}{r}
$$

As Equation 16 cannot be resolved analytically, a numerical solution must be obtained. It can therefore be re-written in incremental terms as follows:

$$
\Delta r=\frac{\Delta \sigma_{r} \cdot r}{2 \cdot \sigma_{c i} \cdot\left(m_{b r e s} \cdot \frac{\sigma_{r}}{\sigma_{c i}}+s_{r e s}\right)^{a_{r e s}}}
$$

The numerical procedure (finite difference method) foresees the division of the plastic zone around the sphere into concentric cortexes and the following calculation steps: $\sigma_{\mathrm{r}}$ is increased by a very small $\Delta \sigma_{\mathrm{r}}$ value for aninternal pressure value $\sigma_{R}=0$, starting from the hemispherical surface $(r=R)$. From the new calculated radial stress, it is then possible, utilizing Equation 17, to determine the increment of radius $r$ and associate this value to the radial stress previously obtained. It is then necessary to proceed in this way until $\sigma_{\mathrm{r}}$ reaches the value $\sigma_{\mathrm{Rpl}}$ (Equation $10 \mathrm{~b}$ ); at this point, the associated radius corresponds to the searched for plastic radius of the rock mass.

\section{Results}

The formulations reported in section 2 have been used to develop a parametric analysis, which has been conducted while varying all the parameters of influence in the ambit of typical tunnel variability intervals. In particular, nine different types of rock masses in a GSI interval of between 30 and 90 and 186 types of soil characterized by different pairs of strength parameter values $\left(c_{p}, \varphi_{p}\right)$ have been considered. Moreover, 4 different compression strength values of intact rock $\left(\sigma_{\mathrm{ci}}=25,55,85\right.$, $115 \mathrm{MPa})$ and 3 different types of $\mathrm{D}$ parameters $(\mathrm{D}=0,0.5$, 1) have also been considered for the studied rock masses (Hoek et al., 2002). The parameter $\mathrm{m}_{\mathrm{i}}$ proved to be not of influence and was therefore made equal to an intermediate value (16) of its typical field of variability (6-26) (Hoek et al., 2002). The parametric study for the rock masses was conducted considering 252 cases.

The maximum lithostatic stress value $\mathrm{p}_{0}$ for which no plastic zone formed around the hemispheric surface, that is, the limit stability condition of the excavation face due to the absence of a plastic zone ahead of it, was obtained for all the analyzed cases. This $p_{0 \max }$ value resulted to be independent of the tunnel radius and it was diagrammed for different GSI values (rock masses) and for different cohesion values $\mathrm{c}_{\mathrm{p}}$ (soils).

The results of the parametric study are reported in Fig. 3 (soils) and in Fig. 4-5 (rock masses).

\section{Discussion}

From an analysis of the figure it is possible to note that, for the different types of soil, $\mathrm{p}_{0 \max }$ increases linearly with the cohesion. Moreover, for each specific cohesion value, $p_{0 \max }$ also increases almost linearly in function of the friction angle. The plasticization of the ground at the border of the hemisphere, which represents the excavation face, occurs for modest depths (at about $30 \mathrm{~m}$ for ground with a high friction angle and at about $20 \mathrm{~m}$ for ground with a low friction angle) in average cohesive soil, i.e., $\mathrm{c}_{\mathrm{p}}=0.2 \mathrm{MPa}$.

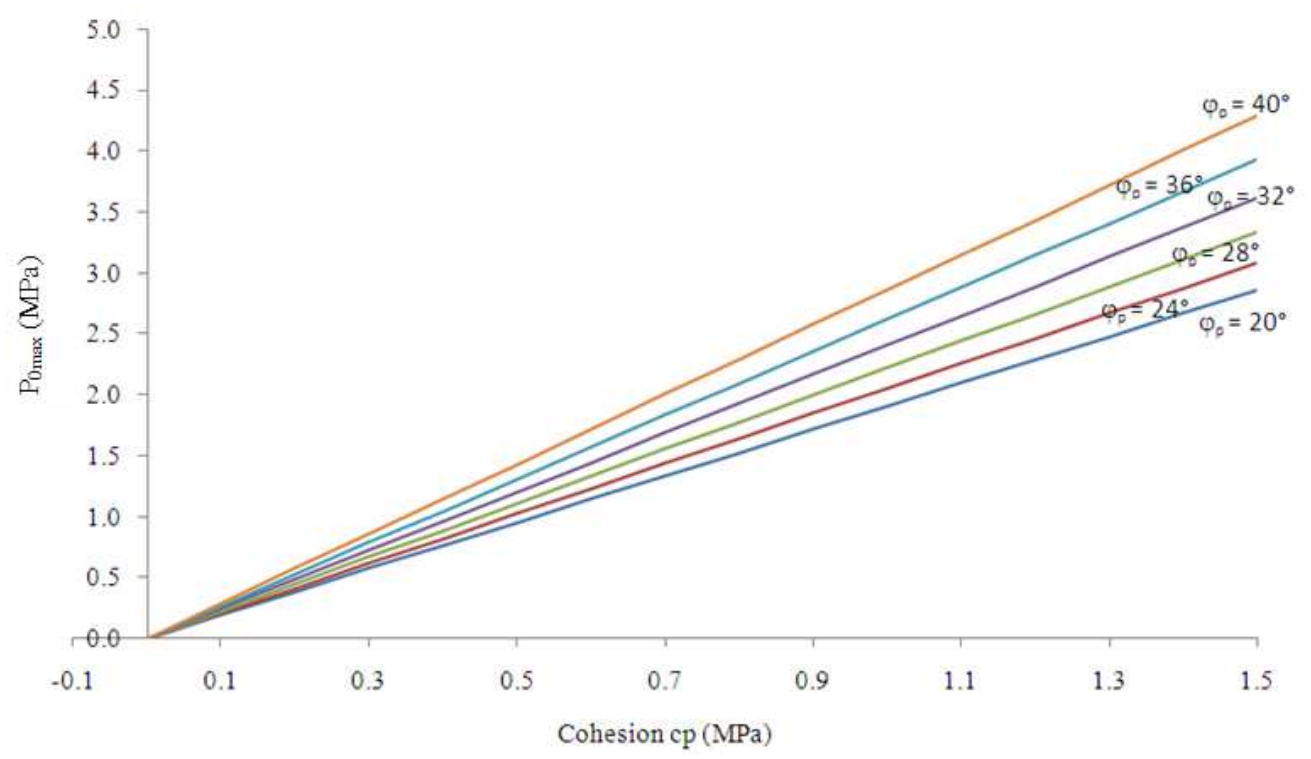

Fig. 3. Trend of the maximum lithostatic stress $p_{0}$ that can guarantee the absence of a plastic zone at the border of the hemisphere which represents the excavation face, with variations of the peak cohesion of the soil, for different peak friction angles 


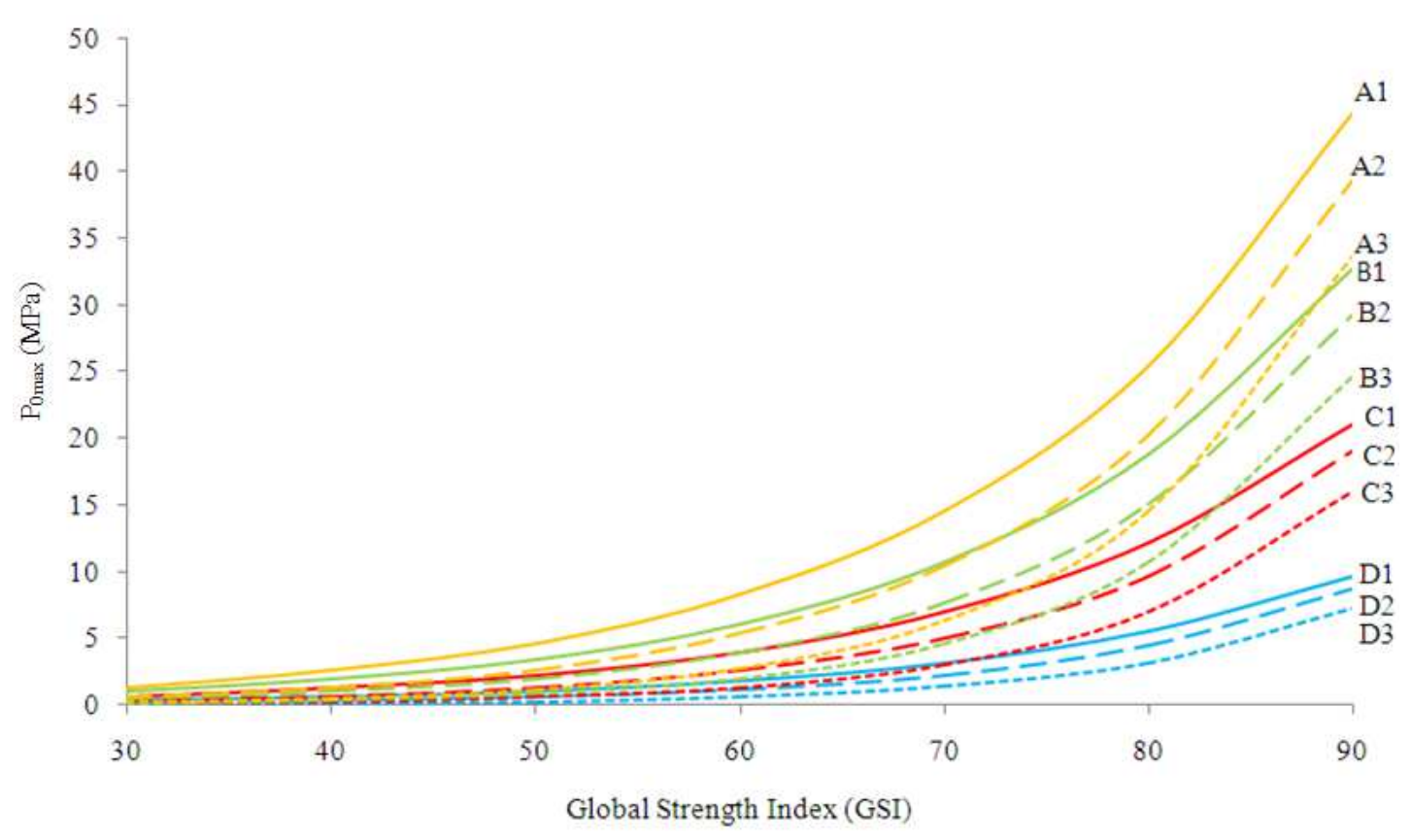

Fig. 4. Trend of the maximum lithostatic stress $\mathrm{p}_{0}$ that can guarantee the absence of a plastic zone at the edge of the hemisphere which represents the excavation face, with variations of the GSI of the rock mass, for different uniaxial compression strength values of the intact rock $\left(\sigma_{\mathrm{ci}}\right)$ and of parameter D (disturbance factor). Key: (A) $\sigma_{\mathrm{ci}}=115 \mathrm{MPa}$; (B) $\sigma_{\mathrm{ci}}=85 \mathrm{MPa}$; (C) $\sigma_{\mathrm{ci}}=55$ $\mathrm{MPa} ;(\mathrm{D}) \sigma_{\mathrm{ci}}=25 \mathrm{MPa}$; (1) $\mathrm{D}=0$; (2) $\mathrm{D}=0,5 ;(3) \mathrm{D}=1$

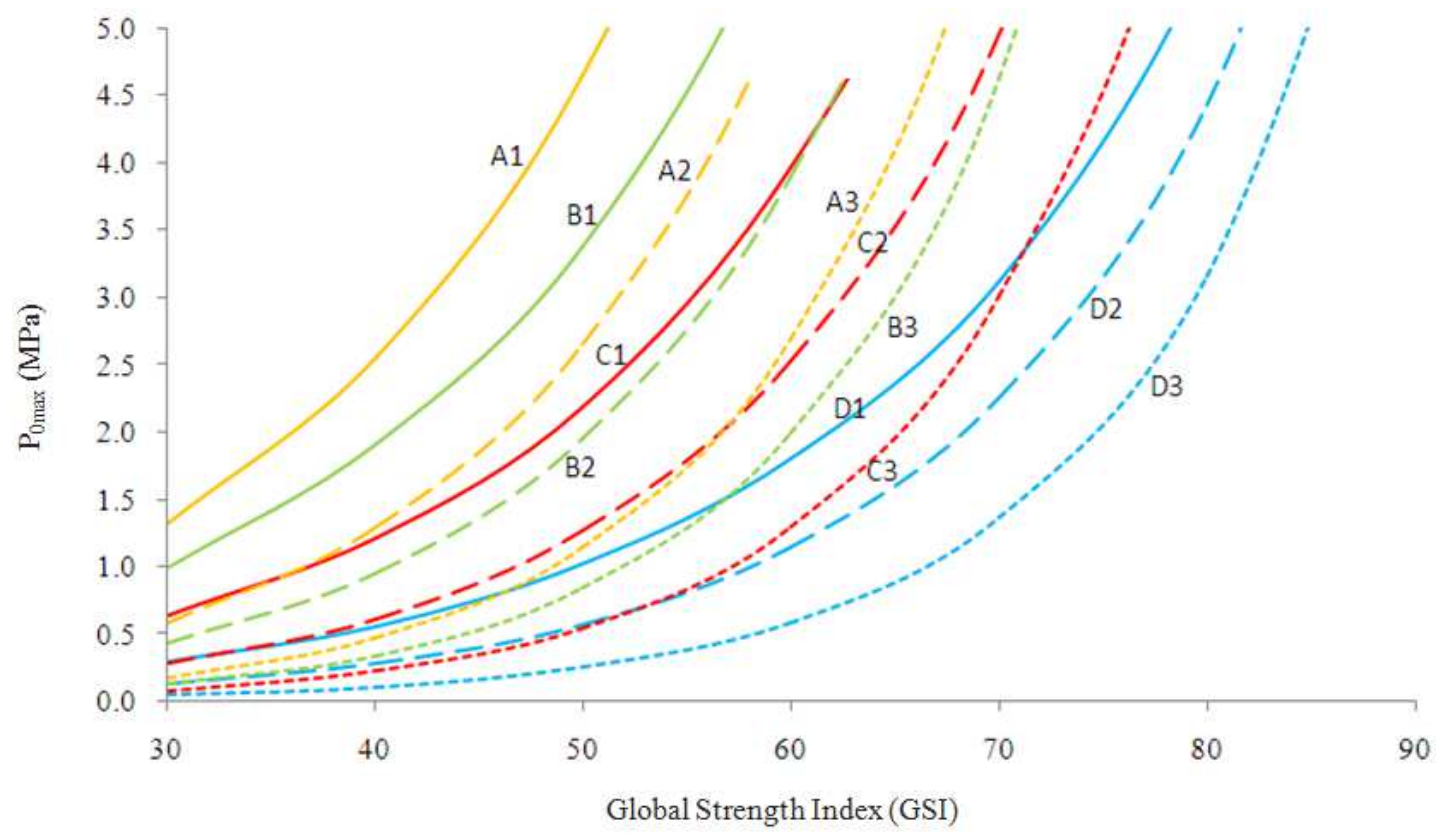

Fig. 5. Details of Fig. 4. Key: (A) $\sigma_{\mathrm{ci}}=115 \mathrm{MPa}$; (B) $\sigma_{\mathrm{ci}}=85 \mathrm{MPa}$; (C) $\sigma_{\mathrm{ci}}=55 \mathrm{MPa}$; (D) $\sigma_{\mathrm{ci}}=25 \mathrm{MPa}$; (1) $\mathrm{D}=0$; (2) $\mathrm{D}=0,5 ;$; (3) $\mathrm{D}=1$

Instead, in rock masses, $\mathrm{p}_{0 \max }$ grows with a similar trend to that of a parabola as the GSI is varied. Greater values were observed for higher intact rock strength values and for lower disturbance factor $\mathrm{D}$ values. The relation between $\mathrm{p}_{0 \max }$ and $\sigma_{\mathrm{ci}}$ is almost linear for a given GSI and the same D value. The $\mathrm{D}$ parameter produces a percentage reduction of $\mathrm{p}_{0 \max }$ with respect to the $\mathrm{D}=0$ case, regardless of the GSI or $\sigma_{\mathrm{ci}}$. The excavation face in rock masses with an elevated GSI and $\sigma_{\mathrm{ci}}$ can still be stable at depths of $1500 \mathrm{~m}$ (that is, for $\mathrm{p}_{0}$ almost equal to $45 \mathrm{MPa}$ ). 


\section{Conclusion}

The stability of an excavation face is one of the most important aspects in the design of a tunnel. In the case in which the excavation face is not naturally stable, it is necessary to carry out reinforcement interventions, such as doweling with fiberglass bars. Because of the elevated cost of reinforcements, these operations should only be introduced after establishing the instability of the excavation face in natural conditions.

The analyses of the stability conditions of the excavation face can be conducted, with a certain degree of detail, through numerical modelling. An approximate analytical method that allows a rough idea to be obtained in a short time for deep tunnels is the convergenceconfinement method in which a spherical geometry is used to represent the excavation face.

In this study, after having presented the most important relations that allow the stress state at the border of a hemisphere to be described for the case in which the strength criterion is of a linear type, a numerical solution is presented to describe the stress state and the plastic radius in rock masses in which the strength criterion is curved (Hoek-Brown strength criterion).

An extended parametric analysis has been conducted for different types of soil and rock masses and this has allowed a maximum lithostatic value $p_{0 \max }$ to be obtained which still allows the absence of a plastic zone around the hemisphere for variations of the geometric and geotechnical parameters that condition the problem. This $\mathrm{p}_{0 \max }$ value was then plotted in function of the cohesion (soils) and of the GSI (rock masses).

The reported graphs make it possible to obtain a preliminary estimation of the maximum depth of the tunnel where no plastic zone occurs at the border of the hemisphere which, with a certain degree of approximation, represents the excavation face. However, a more detailed evaluation would require the use of axial symmetric bi-dimensional or even three-dimensional numerical modelling.

\section{Acknowledgement}

This research was performed with use of the equipment of the DIATI Department of Politecnico di Torino.

\section{References}

AFTES, 1993. Groupe de travail n.7-Soutenement et revetement, Emploi de la méthode convergenceconfinement, Tunnels et ouvragessouterrains, Supplément au n.117, maj-juin, pp: 118-205.

AFTES, 2001. Recommandations on the convergenceconfinement method.
Anagnostou, G. and K. Kovari, 1996. Face stability conditions with earth-pressure-balanced shields. Tunnelling Underground Space Technol., 11: 165173. DOI: $10.1016 / 0886-7798(96) 00017-X$

Boldini, D., A. Graziani and R. Ribacchi, 2004. Raticosa tunnel, Italy: Characterization of tectonized clayshale and analysis of monitoring data and face stability. Soils Foundations, 44: 57-69.

Carranza-Torres, C. and C. Fairhurst, 2000. Application of the convergence-confinement method of tunnel design to rock masses that satisfy the hoek-brown failure criterion. Tunnelling Underground Space Technol., 15: 187-213.

DOI: $10.1016 / \mathrm{S} 0886-7798(00) 00046-8$

Dias, D. and R. Kastner, 2005, Modélisation numérique de l'apport du renforcement par boulonnage du front de taille des tunnels. Canadian Geotechnical J., 42: 1656-1674. DOI: 10.1139/t05-086

Dias, D., R. Kastner and P. Dubois, 1997. Tunnel face reinforcement by bolting: Strain approach using 3D analysis. Proceedings of the International Conference on Tunneling Under Difficult Conditions, Oct. 27-29, Basel Switzerland, pp: 163-174.

Do, N.A., D. Dias, P. Oreste and I. Djeran-Maigre, 2013. 2D numerical investigation of segmental tunnel lining behavior. Tunnelling Underground Space Technol., 37: 115-127.

Do, N.A., D. Dias, P. Oreste and I. Djeran-Maigre, 2014a. A new numerical approach to the hyperstatic reaction method for segmental tunnel linings. Int. J. Numerical Analytical Methods Geomechan., 38: 1617-1632. DOI: 10.1002/nag.2277

Do, N.A., D. Dias, P. Oreste and I. Djeran-Maigre, 2014b. Three-dimensional numerical simulation for mechanized tunnelling in soft ground: The influence of the joint pattern. Acta Geotechnica, 9: 673-694. DOI: 10.1007/s11440-013-0279-7

Do, N.A., D. Dias, P. Oreste and I. Djeran-Maigre, 2014c. Three-dimensional numerical simulation of a mechanized twin tunnels in soft ground. Tunnelling Underground Space Technol., 42: 40-51. DOI: 10.1016/j.tust.2014.02.001

Hoek, E. and E.T. Brown, 1980. Underground Excavations in Rock. 1st Edn., Institution of Mining and Metallurgy, London, pp: 527.

Hoek, E., C. Carranza-Torres and B. Corkum, 2002. Hoek-Brown failure criterion-2002 Edition. Proc. NARMS-TAC Conference, Toronto, 1: 267-273.

Hoek, E., T.G. Carter and M.S. Diederichs, 2013. Quantification of the geological strength index chart. Proceedings of the 47th US Rock Mechanics/Geomechanics Symposium, Jun. 23-26, San Francisco, CA, USA, pp: 1-8.

Kamata, H. and H. Mashimo, 2003. Centrifuge model test of tunnel face reinforcement by bolting. Tunnelling Underground Space Technol., 18: 205212. DOI: $10.1016 / \mathrm{S} 0886-7798(03) 00029-4$ 
Kirsch, A., 2010. Experimental investigation of the face stability of shallow tunnels in sand. Acta Geotechnica, 5: 43-62.

DOI: $10.1007 / \mathrm{s} 11440-010-0110-7$

Leca, E. and L. Dormieux, 1990. Upper and lower bound solutions for the face stability of shallow circular tunnels in frictional material, Geotechnique, 40: 581-606. DOI: 10.1680/geot.1990.40.4.581

Leca, E. and M. Panet, 1988. Application duCalcul a la rupture a la stabilite du front de taille d'un tunnel. Revue Francaise de Geotechnique, 43: 5-19.

Lembo-Fazio, A. and R. Ribacchi, 1986. Stato di sforzo e deformazione intorno ad una galleria. I Conferenza di Meccanica ed Ingegneria delle rocce, Torino Italy.

Lunardi, P. and R. Bindi, 2004. The evolution of reinforcement of the advance core using fibre-glass elements. Felsbau, 22: 8-19.

Lunardi, P., 2008. Design and Construction of Tunnels: Analysis of Controlled Deformations in Rock and Soils (ADECO-RS). 1st Edn., Springer Science and Business Media, Berlin, ISBN-10: 3540738754, pp: 576.

Marinos, P. and E. Hoek, 2000. GSI: A geologically friendly tool for rock mass strength estimation. Proceedings of the International Conference on Geotechnical and Geological Engineering, Nov. 1924, Melbourne, Austrelia.

Marinos, V., P. Marinos and E. Hoek, 2005. The geological strength index: Applications and limitations. Bull Eng. Geol. Environ., 64: 55-65. DOI: $10.1007 / \mathrm{s} 10064-004-0270-5$

Ng, C.W.W. and G.T.K. Lee, 2002. A three-dimensional parametric study of the use of soil nails for stabilising tunnel faces, Computers and Geotechnics, 29: 673697. DOI: 10.1016/S0266-352X(02)00012-5

Oreste, P., 2005. A probabilistic design approach for tunnel supports. Comput. Geotechn., 32: 520-534. DOI: 10.1016/j.compgeo.2005.09.003

Oreste, P., 2005. Back-analysis techniques for the improvement of the understanding of rock in underground constructions. Tunnelling Underground Space Technol., 20: 7-21.

DOI: $10.1016 /$ j.tust.2004.04.002

Oreste, P., 2008. Distinct analysis of fully grouted bolts around a circular tunnel considering the congruence of displacements between the bar and the rock. Int. J. Rock Mechanics Mining Sci., 45: 1052-1067. DOI: $10.1016 /$ j.ijrmms.2007.11.003

Oreste, P., 2013. Face stabilization of deep tunnels using longitudinal fibreglass dowels. Int. J. Rock Mechanics Mining Sci., 58: 127-140.

Oreste, P.P. and D. Dias, 2012. Stabilisation of the excavation face in shallow tunnels using fibreglass dowels. Rock Mechanics Rock Eng., 45: 499-517. DOI: $10.1007 / \mathrm{s} 00603-012-0234-1$
Oreste, P.P., 2003a. Analysis of structural interaction in tunnels using the convergence-confinement approach. Tunnelling Underground Space Technol., 18: 347-363. DOI: 10.1016/S0886-7798(03)00004-X

Oreste, P.P., 2003b. A procedure for determining the reaction curve of shotcrete lining considering transient conditions. Rock Mechanics Rock Eng., 36: 209-236.

Oreste, P.P., 2007. A numerical approach to the hyperstatic reaction method for the dimensioning of tunnel supports. Tunnelling Underground Space Technol., 22: 185-205.

DOI: $10.1016 /$ j.tust.2006.05.002

Oreste, P.P., 2009. Face stabilisation of shallow tunnels using fibreglass dowels. Proc. Institution Civil Eng. Geotechnical Eng., 162: 95-109. DOI: 10.1680/geng.2009.162.2.95

Osgoui, R.R. and P. Oreste, 2010. Elasto-plastic analytical model for the design of grouted bolts in a Hoek-Brown medium. Int. J. Numerical Analytical Methods Geomechan., 34: 1651-1686. DOI: $10.1002 /$ nag. 823

Panet, M. and A. Guenot, 1982. Analysis of convergence behind the face of a tunnel. Proc. Tunnelling Brighton, 82: 197-204.

Panet, M., 1995. Le calcul des tunnels par la méthode convergence-confinement, Presses de l'écolenationale des Ponts et chaussées, Paris.

Rechsteiner, G.F. and G. Lombardi, 1974. Unemethode de calculelasto-plastique de l'etatde tension et de deformation autourd'unecavitesouterraine. Proceedings of the 3rd Congress of the International Society for Rock Mechanics, (SRM' 74), pp: 1049-1054.

Ribacchi, R. and R. Riccioni, 1977. Stato di sforzo e deformazione intorno ad una galleria circolare. Gallerie e Grandi Opere Sotterranee, 5: 7-18.

Wong, H., D. Subrin and D. Dias, 2000. Extrusion movements of a tunnel head reinforced by finite length bolts-a closed-form solution using homogenization approach. Int. J. Numerical Analytical Methods Geomechan., 24: 533-565. DOI: 10.1002/(SICI)1096-9853(200005)24:6<533::AIDNAG80>3.0.CO;2-8

Wong, H., D. Subrin and D. Dias, 2006. Convergenceconfinement analysis of a bolt-supported tunnel using the homogenization method. Canadian Geotechnical J., 43: 462-483. DOI: $10.1139 / \mathrm{t} 06-016$

Wong, H., V. Trompille and D. Dias, 2004. Extrusion analysis of a bolt-reinforced tunnel face with finite ground-bolt bond strength. Canadian Geotechnical J., 41: 326-341.

Yoo, C. and H.K. Shin, 2003. Deformation behaviour of tunnel face reinforced with longitudinal pipeslaboratory and numerical investigation. Comput. Geotechnics, 29: 73-94. 
Pierpaolo Oreste / American Journal of Applied Sciences 2014, 11 (12): 1995-2003 DOI: 10.3844/ajassp.2014.1995.2003

Yoo, C., 2002. Finite-element analysis of tunnel face reinforced by longitudinal pipes. Comput. Geotechnics, 29: 73-94.

DOI: $10.1016 / \mathrm{S} 0266-352 \mathrm{X}(01) 00020-9$
Yu, H.S., 2000. Cavity Expansion Methods in Geomechanics. 1st Edn., Springer Science and Business Media, Dordrecht, ISBN-10: 0412799901, pp: 385. 